\title{
NOVEL CCI-OTA-BASED GROUNDED CAPACITOR CURRENT-MODE BIQUADRATIC BANDPASS AND LOWPASS FILTERS
}

\author{
MUHAMMAD TAHER ABUELMA'ATTI \\ King Fahd University of Petroleum and Minerals, Box 203 Dhahran 31261, Saudi Arabia \\ (Received October 9, 1993; in final form December 15, 1993)
}

\begin{abstract}
A novel current-mode active filter topology using a first generation current conveyor (CCI) and an operational transconductance amplifier (OTA) is presented. Using this topology, grounded-capacitor biquadratic bandpass and lowpass filter sections, with at most five one-port passive elements, are realized. In these realizations, the parameter $\omega_{0}$ can be adjusted without disturbing the parameter $\omega_{0} /$ $\mathrm{Q}_{\text {.. }}$
\end{abstract}

\section{INTRODUCTION}

In active- $\mathrm{RC}$ circuits, it is highly desirable to have all the capacitors grounded [18] for several reasons. First, grounded capacitors are suitable for monolithic IC technology [9-11] and thin film fabrication [2]. In thin film fabrication, if the capacitors are grounded, the etching process is eliminated and the number of contacts is reduced. Also, the parasitic capacitors surrounding the capacitors can be easily accounted for or tuned out as they are now in parallel with the grounded capacitors [3]. Secondly, in CMOS technology, the use of grounded capacitors is an advantage as the bottom plate parasitic capacitor is eliminated altogether and the top plate parasitic capacitor can be accounted for easily as it becomes parallel to the main capacitance [12]. This explains the considerable interest in designing active- $\mathrm{RC}$ circuits with grounded capacitors $[1,3,5,6,13]$.

On the other hand, at present there is a growing interest in designing analog current-mode signal processing circuits. This is attributed to the higher bandwidth, greater linearity, and larger dynamic range obtainable compared to their voltagemode counterparts. The current-conveyor (CC), a powerful analog building block with a current-mode capability and the operational transconductance amplifier (OTA) is basically a differential voltage-controlled current source. CCs are, therefore, highly potential candidates for implementing current-mode filter sections, and recently a number of realizations using the first-generation current-conveyor $(\mathrm{CCI})$, the second-generation current-conveyor (CCII), and the OTA have been presented [14-25].

In most of the CC-based realizations, bandpass and lowpass biquadratic filters require at least two capacitors; one of them floating [14-19]. Moreover, in most of these realizations, the output current exhibiting bandpass/lowpass realization 
has to be taken out through another $\mathrm{CC}$. Thus, at least two active elements are required. In some CC-based realizations, bandpass and lowpass biquadratic filters are realized using at least two grounded capacitors [20-24]. The circuit proposed by Fabre et al [20] requires four, one-port passive RC elements; two grounded capacitors and two resistors, and two CCs. An additional CC is required to take out the output current. Moreover, the CCs are different; one CCI and the other CCII. The circuit proposed by Chang [21] requires six one-port passive RC elements; two grounded capacitors and four resistors, and one CCII. An additional $\mathrm{CC}$ is also required to take out the output current. The circuit proposed by Cang and Chen [22] requires eight one-port passive EC elements; two grounded capacitors and six resistors, and five CCIIs. The circuit proposed by Chang and Chen [23] requires eight one-port passive RC elements; three grounded capacitors and five resistors, and five CCIIs. The circuit proposed by Senani [24] requires six passive one-port elements; two grounded capacitors and four grounded resistors, two $\mathrm{CCII}-$, four $\mathrm{CCI}-$ and one $\mathrm{CCII}+$.

The OTA-based bandpass and lowpass realizations [25] requires three grounded one-port passive elements; two capacitors and one resistor, and five OTAs.

In the available literature no attempt has been reported to combine $\mathrm{CC}$ and OTA in a current-mode biquadratic filter realization.

It is the major intention of this paper to present a CCI-OTA-based low-component current-mode lowpass and bandpass biquadratic filter realizations. The proposed realizations use at most five one-port passive elements; two (or three) grounded capacitors and three (or two) resistors, one CCI + and one OTA.

\section{PROPOSED CIRCUIT}

Consider the circuit shown in Fig. 1. Assuming ideal CCI and OTA with

$\mathrm{I}=\mathrm{g}_{\mathrm{m}}\left(\mathrm{V}^{+}-\mathrm{V}^{-}\right), \quad \mathrm{I}_{\mathrm{x}}=\mathrm{I}_{\mathrm{y}}=\mathrm{I}_{\mathrm{z}}, \quad \mathrm{V}_{\mathrm{x}}=\mathrm{V}_{\mathrm{y}}$

routine analysis yields

$\frac{I_{o}}{I_{i}}=\frac{Z_{2} Z_{3}\left(1+g_{m} Z_{1}\right)}{Z_{3}\left(Z_{1}+Z_{2}+g_{m} Z_{2} Z_{4}\right)-Z_{4}\left(Z_{1}+Z_{2}+Z_{3}\right)}$

where the current $I_{o}=-I_{z}$ is directly obtained through the z-port of the CCI.

In the following subsections it will be shown that bandpass (BP) and lowpass (LP) biquadratic filter transfer functions can be obtained from eqn. (1).

\subsection{Bandpass Realization}

If we choose

$$
\mathrm{Z}_{1}=\mathrm{R}_{1}, \quad \mathrm{Z}_{2}=\frac{1}{\mathrm{sC}_{2}}, \quad \mathrm{Z}_{3}=\infty, \quad \mathrm{Z}_{4}=\frac{1}{\mathrm{sC}_{4}}
$$




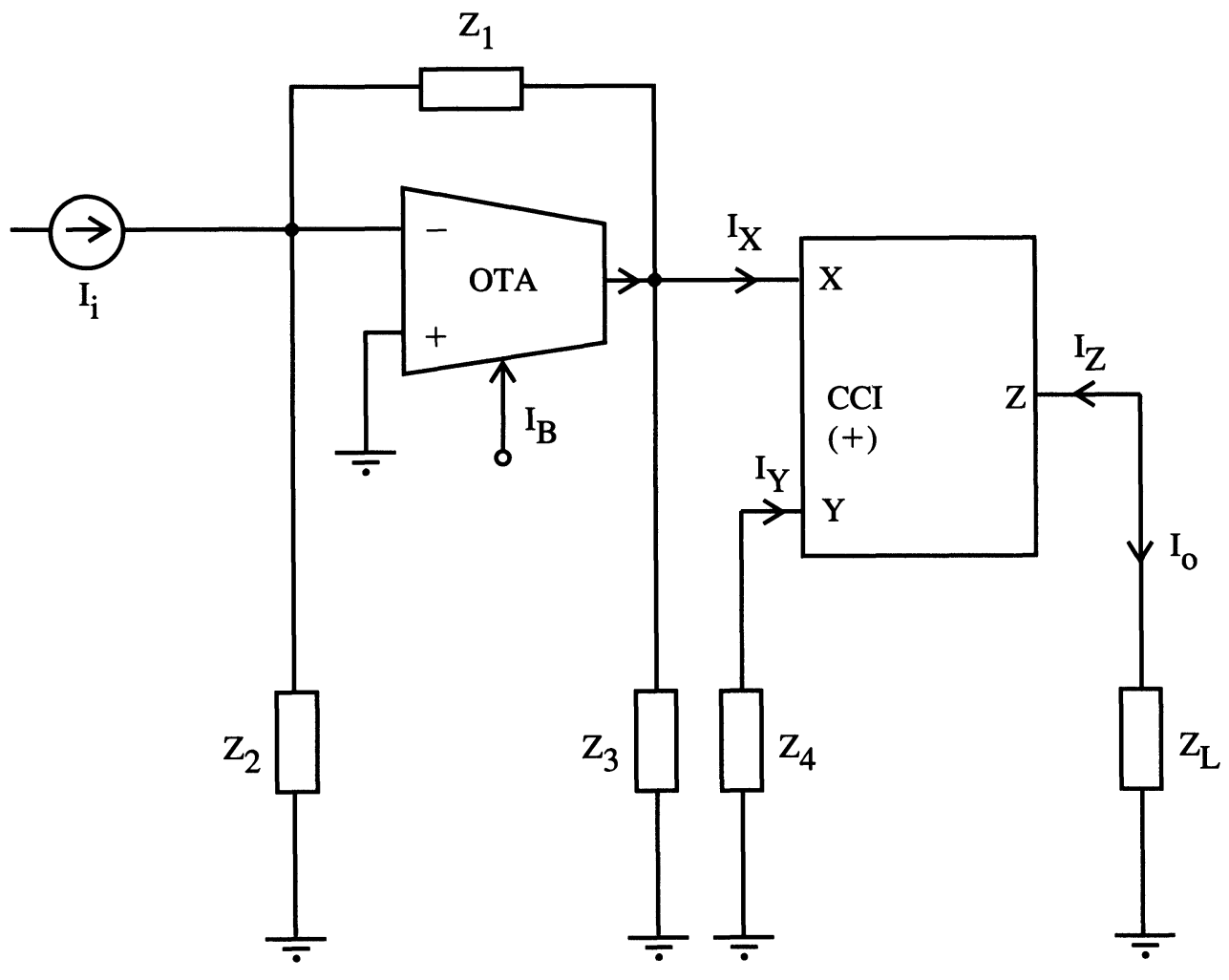

FIGURE 1 Proposed current-mode achre filter using OTA and CCI.

Eqn. (1) reduces to

$H_{1}(s)=\frac{I_{o}}{I_{i}}=\frac{s_{4}\left(1+g_{m} R_{1}\right)}{g_{m}+s\left(C_{4}-C_{2}\right)+s^{2} C_{2} C_{4} R_{1}}=\frac{\alpha s}{s^{2}+\frac{\omega_{0}}{Q_{o}} s+\omega_{0}^{2}}$

which corresponds to the transfer function of a bandpass filter with the parameters

$\omega_{\mathrm{o}}^{2}=\frac{\mathrm{g}_{\mathrm{m}}}{\mathrm{C}_{2} \mathrm{C}_{4} \mathrm{R}_{1}}$

$\alpha=\frac{1+\mathrm{g}_{\mathrm{m}} \mathrm{R}_{1}}{\mathrm{C}_{2} \mathrm{R}_{1}}$

and

$\frac{\omega_{0}}{Q_{0}}=\frac{C_{4}-C_{2}}{C_{2} C_{4} R_{1}}$ 
This bandpass filter realization requires only three one-port passive elements; two grounded capacitors and one resistor. Also, the parameter $\omega_{0}$ can be tuned by adjusting $g_{m}$ without disturbing the parameter $\omega_{0} / Q_{0}$. By choosing $C_{4}>>C_{2}$ it is easy to show that the sensitivities of $\omega_{0}$ and $\omega_{0} / Q_{0}$ to the active and passive components are given by

$\left|S_{\mathbf{x}_{\mathrm{i}}}^{\omega_{0}}\right|=\left|\mathbf{S}_{\mathbf{x}_{\mathrm{i}}}^{\omega_{v_{i}} / \mathbf{Q}_{0}}\right|=\frac{1}{2}$

\subsection{Bandpass and Lowpass Realizations}

If we choose

$\mathrm{Z}_{1}=\infty, \quad \mathrm{Z}_{2}=\mathrm{R}_{2}, \quad \mathrm{Z}_{3}=\frac{\mathrm{R}_{3}}{1+\mathrm{sC}_{3} \mathrm{R}_{3}}, \quad \mathrm{Z}_{4}=\mathrm{R}_{4}+\frac{1}{\mathrm{sC}_{4}}$

eqn. (1) reduces to

$$
\begin{aligned}
H_{2}(s) & =\frac{I_{o}}{I_{i}}=\frac{-s C_{4} g_{m} R_{2} R_{3}}{1+s\left(C_{3} R_{3}+C_{4} R_{4}-C_{4} R_{3}\right)+s^{2} C_{3} C_{4} R_{3} R_{4}} \\
& =\frac{\beta s}{s^{2}+\frac{\omega_{0}}{Q_{0}} s+\omega_{o}^{2}}
\end{aligned}
$$

which corresponds to the transfer function of a bandpass filter with the parameters

$$
\begin{aligned}
& \omega_{o}^{2}=\frac{1}{C_{3} C_{4} R_{3} R_{4}} \\
& \beta=\frac{g_{m} R_{2}}{C_{3} R_{4}} \\
& \frac{\omega_{0}}{Q_{0}}=\frac{C_{3} R_{3}+C_{4} R_{4}-C_{4} R_{3}}{C_{3} R_{3} C_{4} R_{4}}
\end{aligned}
$$

If we choose $R_{3}=R_{4}$ eqn. (9) reduces to

$\frac{\omega_{0}}{Q_{0}}=\frac{1}{C_{4} R_{4}}$

This bandpass filter realization requires five one-port passive elements; two grounded capacitors and three resistors. From eqn. (7) and eqn. (10), it is obvious that the parameter $\omega_{0}$ can be tuned by adjusting $C_{3}$ without disturbing the parameter $\omega_{0} / Q_{0}$. The gain of this bandpass realization can be adjusted by changing the parameter $g_{m}$ and/or the resistance $R_{2}$ without disturbing the parameters $\omega_{0}$ and $\omega_{0} / Q_{0}$. It is easy to show that the sensitivities of the parameters $\omega_{0}$ and $\omega_{0} / Q_{0}$ to passive components are low. 
If we choose $Z_{2}=1 / s C_{2}$ instead of $Z_{2}=R_{2}$, eqn. (1) reduces to

$$
\begin{aligned}
H_{3}(s) & =\frac{I_{0}}{I_{i}}=\frac{C_{4} g_{m} R_{3} / C_{2}}{1+s\left(C_{3} R_{3}+C_{4} R_{4}-C_{4} R_{3}\right)+s^{2} C_{3} C_{4} R_{3} R_{4}} \\
& =\frac{\gamma}{s^{2}+\frac{\omega_{0}}{Q_{0}} s+\omega_{o}^{2}}
\end{aligned}
$$

which corresponds to the transfer function of a lowpass filter with the parameters $\omega_{0}^{2}$ and $\omega_{0} / Q_{0}$ the same as in eqn. (7) and eqn. (8) and the parameter $\gamma$ is given by

$\gamma=\frac{\mathrm{C}_{4}}{\mathrm{C}_{2}} \mathrm{~g}_{\mathrm{m}} \mathrm{R}_{3}$

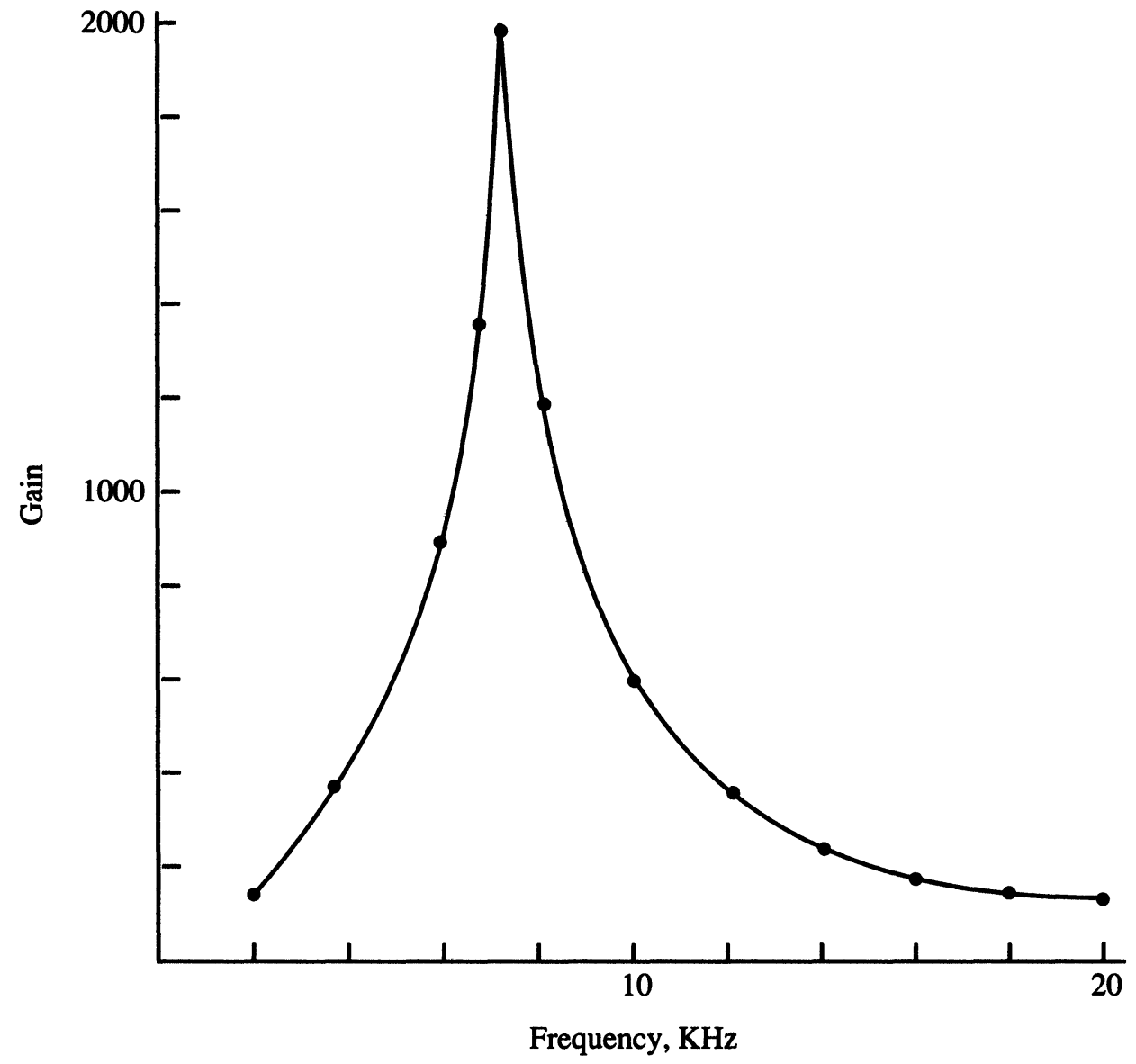

FIGURE 2 Characteristics of the BPF of Eqn. (2) : Calculated (eqn. (2)), •: Simulated. 
This lowpass filter realization requires three grounded capacitors and two resistors. By selecting $R_{3}=R_{4}$ or $C_{3}=C_{4}$, it is easy to see that the parameters $\omega_{0}^{2}$ and $\omega_{0} /$ $\mathrm{Q}_{\mathrm{o}}$ are not interdependent; that is, the parameter $\omega_{0}^{2}$ can be tuned without disturbing the parameter $\omega_{0} / Q_{0}$. The gain of this lowpass realization can be adjusted by changing the parameter $\mathrm{g}_{\mathrm{m}}$ and/or the capacitance $\mathrm{C}_{2}$ without disturbing the parameters $\omega_{0}$ and $\omega_{0} / Q_{0}$.

\section{SIMULATION RESULTS}

All the current transfer functions presented in this paper have been verified using IS-SPICE simulations based on the translinear CC implementations of Fabre et al. [20]. Fig. 2 shows the simulated and calculated current transfer functions of the bandpass filter realization of eqn. (2). From Fig. 2, it is obvious that the agreement between simulated and calculated results appears to be good.

\section{CONCLUSION}

In this paper a new topology for realizing current mode bandpass and lowpass biquadratic filters has been presented. This topology uses an operational transconductance amplifier, a first generation current conveyor, and five one-port passive elements at most. In the resulting lowpass and bandpass realizations, the parameters $\omega_{0}^{2}$ can be tuned without disturbing the parameter $\omega_{0} / Q_{0}$. In one of the realizations, the gain of the lowpass and bandpass filters can be adjusted without disturbing the other parameters of the filters. The proposed lowpass and bandpass realizations enjoy low active and passive sensitivities. In all the proposed realizations, the capacitors are grounded; this is an attractive feature for integration.

\section{REFERENCES}

1. R.S. Sidorowicz, An abundance of sinusoidal RC oscillators, Proceedings IEE, Vol. 119, 1972, pp. 283-293.

2. M.S. Abougabal, B.B. Battacharyya, and M.N.S. Swamy, An optimal design of RC active filters using grounded capacitors, International Journal of Circuit Theory and Applications, Vol. 6, 1978, pp. 31-40.

3. R.S. Sidorowicz, Some novel RC oscillators for radio frequencies, Electronic Engineering, Vol. 39,1967 , pp. 498-502, 560-564.

4. L.P. Heulsman, Equal-valued-capacitor active RC network realisation of a third-order lowpass Butterworth characteristic, Electronics Letters, Vol. 7, 1971, pp. 271-271.

5. M. Sundramurthy, B.B. Battacharyya and M.N.S. Swamy, A simple voltage controlled oscillator with grounded capacitors, Proceedings IEEE, Vol. 65, 1977, pp. 1612-1614.

6. B.B. Battacharyya, M. Sundramurthy and M.N.S. Swamy, Realisation of tunable RC-active oscillators using grounded capacitors and voltage amplifiers, International Journal of Circuit Theory and Applications, Vol. 8, 1980, pp. 355-371.

7. R.A. Stein, Synthesis of grounded-capacitor multiport RC networks, IEEE International Symposium on Circuit Theory, 1971, pp. 49-50.

8. M.S. Abougabal, B.B. Battacharyya, Easily integrable very-low-sensitivity active RC filter, Electronics Letters, Vol. 8, 1972, pp. 303-304. 
9. G.S. Moschytz and W. Thelen, Design of hybrid integrated-filter building blocks, IEEE Journal of Solid State Circuits, Vol. SC-5, 1970, pp. 99-107.

10. G.S. Moschytz, Gain-sensitivity product-a figure of merit for hybrid-integrated filters using single operational amplifiers, IEEE Journal of Solid State Circuits, Vol. SC-6, 1971, pp. 103-110.

11. R.W. Newcomb, Active integrated synthesis, Prentice-Hall, New Jersey, 1968.

12. R. Senani, Simple sinusoidal oscillator using OpAmp compensation poles, Electronics Letters, Vol. 29, 1993, pp. 452-453.

13. M.T. Darkani and B.B. Battacharyya, Generation and design of canonic grounded-capacitor variable-frequency RC-active oscillators, IEE Proceedings, Vol. 132, Part G, 1985, pp. 153-160.

14. S.-I. Liu, H.-W. Tsao and J. Wu, Cascadable current-mode single CCII biquads, Electronics Letters, Vol. 26, 1990, pp. 2005-2006.

15. C.-M. Chang, Universal active current filters using single second-generation current conveyor, Electronics Letters, Vol. 27, 1991, pp. 1614-1617.

16. M. Alami and A. Fabre, Insensitive current-mode bandpass filter implemented from two current conveyors, Electronics Letters, Vol. 27, 1991, pp. 897-899.

17. A. Fabre, F. Martin, and M. Hanafi, Current mode allpass/notch and bandpass filters with reduced sensitivities, Electronics Letters, Vol. 26, 1990, pp. 1495-1496.

18. A. Fabre and J.-L. Houle, Voltage-mode and current-mode Sallen-Key implementations based on translinear conveyors, IEE Proceedings-G, Vol. 139, 1992, pp. 491-497.

19. C.-L. Hou, Y.-P. Wu and S.-I. Liu, New configuration for single-CCII first-order and biquadratic current-mode filters, International Journal of Electronics, Vol. 71, 1991, pp. 637-644.

20. A. Fabre and M. Alami, Insensitive current-mode bandpass implementations-based nonideal gyrators, IEEE Transactions on Circuits and Systems-I, Vol. 39, -1992 , pp. 152-155.

21. C.-M. Chang, Current mode allpass/notch and bandpass filter using single CCII, Electronics Letters, Vol. 27, 1991, pp. 1812-1813.

22. C.-M. Cheng and P.-C. Chen, Universal active current filter with three inputs and one output using current conveyors, International Journal of Electronics, Vol. 71, 1991, pp. 817-819.

23. C.-M. Chang and P.-C. Chen, Realization of current-mode transfer function using second-generation current conveyors, International Journal of Electronics, Vol. 71, 1991, pp. 809-815.

24. R. Senani, New current-mode biquad filter, International Journal of Electronics, Vol. 73, 1992, pp. 735-742.

25. C.-M. Chang and P.-C. Chen, Universal active filter with current gain using OTAs, International Journal of Electronics, Vol. 71, 1991, pp. 805-808. 

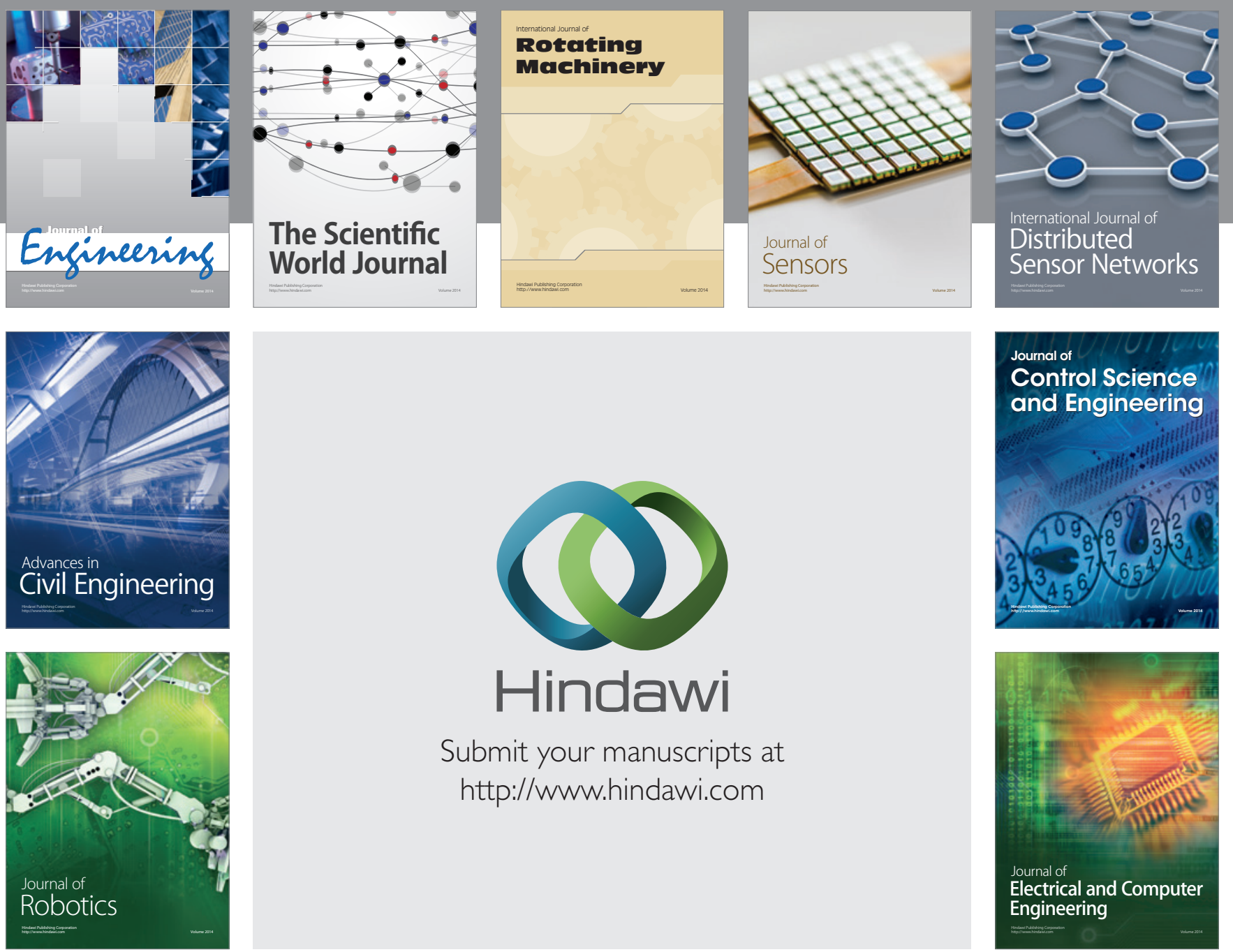

Submit your manuscripts at

http://www.hindawi.com
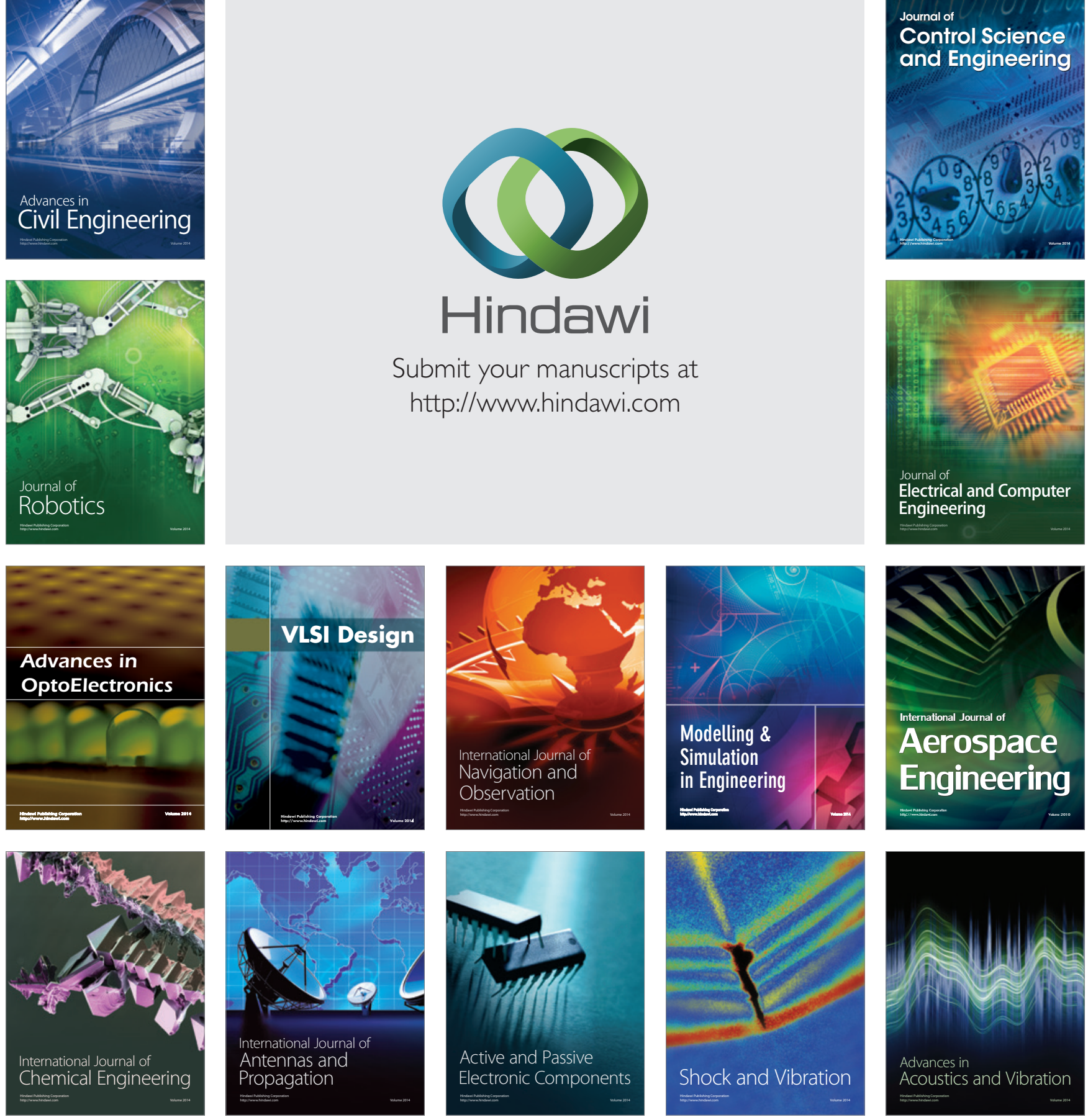